\title{
Neuropathic pain is associated with depressive behaviour and induces neuroplasticity in the amygdala of the rat
}

\author{
Leonor Gonçalves $^{\text {a,b }}$, Rui Silva ${ }^{\text {a }}$, Filipa Pinto-Ribeiro a , José M. Pêgo a , João M. Bessa a , \\ Antti Pertovaara ${ }^{\mathrm{b}}$, Nuno Sousa ${ }^{\mathrm{a}}$, Armando Almeida ${ }^{\mathrm{a}, *}$ \\ a Life and Health Sciences Research Institute (ICVS), School of Health Sciences, University of Minho, Campus de Gualtar, 4710-057 Braga, Portugal \\ ${ }^{\mathrm{b}}$ Department of Physiology, Institute of Biomedicine, University of Helsinki, Helsinki, Finland
}

\section{A R T I C L E I N F O}

\section{Article history:}

Received 22 February 2008

Revised 24 April 2008

Accepted 28 April 2008

Available online $\mathrm{xxxx}$

\section{Keywords:}

Neuropathic pain

Amygdala

Adult neurogenesis

Depressive-like behaviour

\begin{abstract}
A B S T R A C T
Chronic pain is associated with the development of affective disorders but the underlying mechanisms are not fully understood. Changes in brain centres implicated in both emotional and pain processing are likely to be critical in the interplay of pain control and affective emotional behaviour. In the present study, we assessed emotional behaviour and performed a structural analysis of the amygdala (AMY) in neuropathic rats after two months of hyperalgesia and allodynia, induced by the spared nerve injury model (SNI). When compared with Sham-controls, SNI animals displayed signs of depressive-like behaviour. In addition, we found an increased amygdalar volume in SNI rats. No alterations were found in the dendritic arborizations of AMY neurons but, surprisingly, the amygdalar hypertrophy was associated with an increased cell proliferation [bromodeoxyuridine (BrdU)-positive cells] in the central (CeA) and basolateral (BLA) amygdaloid nuclei. The phenotypic analysis of the newly-acquired cells revealed that they co-label for neuronal markers (BrdU + NeuN and BrdU+ Calbindin), but not for differentiated glial cells (BrdU+glial fibrillary acidic protein).

We demonstrate that neuropathic pain promotes generation of new neurons in the AMY. Given the established role of the AMY in emotional behaviour, we propose that these neuroplastic changes might contribute for the development of depressive-like symptoms that are usually present in prolonged pain syndromes in humans.
\end{abstract}

(c) 2008 Elsevier Inc. All rights reserved.

\section{Introduction}

Pain is a multidimensional experience with sensitive-discriminative and motivational-affective dimensions (Anand and Craig, 1996). Persistent pain, including chronic pain syndromes (Tal and Bennett, 1994), is a common condition associated to a wide spectrum of disorders including cancer, inflammation and neuropathic pain. Neuropathic pain (NP) is caused by a primary lesion or dysfunction of the nervous tissue (Merskey and Bogduk, 1994) and results in prolonged hyperalgesia, allodynia and spontaneous pain (Devor, 2006).NP results from a process of peripheral and central sensitization that generates an enhanced transmission of nociceptive input to the brain (Gao et al., 2005; Ren and Dubner, 1996), which may impair the endogenous supraspinal pain control system (Danziger et al., 2001; Kauppila et al., 1998; Pertovaara, 2000; Rasmussen et al., 2004; Tal and Bennett, 1994).

The amygdala (AMY) is a central component of the limbic system and plays a crucial role in behavioural responses to emotional stimuli (Davis and Whalen, 2001; Han and Neugebauer, 2001; Neugebauer and $\mathrm{Li}, 1992)$. Moreover, the AMY is deeply involved in processing the emotional component of pain, probably through a modulatory role

\footnotetext{
* Corresponding author.

E-mail address: aalmeida@ecsaude.uminho.pt (A. Almeida).

URL: http://www.ecsaude.uminho.pt/icvs/domains/neurc/index.htm (A. Almeida).
}

upon major supraspinal pain control centres (SPCC) (Manning and 49 Mayer, 1995; Manning, 1998; Manning et al., 2001). On the other hand, 50 it is possible that neuroplasticity in higher centres controlling SPCC 51 may contribute to alterations in the fine control of pain. In fact, an 52 imbalance between inhibiting and facilitating descending modulation 53 of nociceptive transmission may underlie, at least in part, the 54 development of chronic pain (Almeida et al., 2006; Lima and Almeida, 55 2002; Pertovaara, 2000; Porreca et al., 2002; Schaible et al., 1991). 56 Accordingly, arthritic and neuropathic pain enhance synaptic trans- 57 mission of nociceptive-specific input to the AMY (Han and Neugebauer, 58 2004; Neugebauer and Li, 1992; Neugebauer et al., 2003), which 59 reinforces the potential role of AMY in SPCC alterations resulting from 60 prolonged pain syndromes.

Chronic pain induces mood disorders, including depression and 62 anxiety (Rasmussen, 2004). In addition, the adverseness of pain is am- 63 plified or reduced depending on the emotional environment (Merskey, 64 1965), and conditions of increased anxiety (Rhudy and Meagher, 2000) 65 and depression (Merskey, 1965; Willoughby et al., 2002; Zelman et al., 66 1991) are usually associated with decreased pain tolerance. This vicious 67 circle may trigger, or even result from, neuronal changes in the limbic 68 system. Accordingly, imaging studies indicate that gross structural 69 changes may occur in the AMY in situations of major depression 70 (Altshuler et al., 2005; Bremner et al., 2000; Frodl et al., 2002; Strakowski 71 et al., 1999; Tebartz van Elst et al., 2000). 
As a rationale for the present study, we hypothesized that chronic pain induces emotional disturbances that are associated with neuroplasticity of the amygdaloid complex. To assess this hypothesis, we performed behavioural, stereological and immunocytochemical analysis during or after the induction of a two month neuropathy following the model of Decosterd and Woolf(2000). Part of the present results have already been published in abstract form (Gonçalves et al., 2006).

\section{Materials and methods}

\section{Animals}

All procedures were performed on adult (200-250 g, 55-65 days) male Wistar-Han rats. Animals were housed under standard laboratory conditions ( $12 \mathrm{~h} \mathrm{light}$ cycle; $22^{\circ} \mathrm{C}, 55 \%$ humidity; food and water available ad libitum). Experiments were conducted in accordance with local regulations, European Union Directive 86/609/EEC, NIH guidelines on animal care experimentation and IASP ethical guidelines for pain experimentation on awaken animals (Zimmermann, 1983). Sixty animals were divided in two main experimental groups of 30 rats each: spared nerve injury (SNI) and sham operated (Sham). A set of rats ( $n=18$ each group) received one injection of the cell proliferation marker bromodeoxyuridine (BrdU; Miller and Nowakowski, 1988), $50 \mathrm{mg} / \mathrm{kg}$ body weight, i.p. (Sigma, St. Louis, MO) for three consecutive days before their death (see below), two months after SNI induction or Sham surgery.

Spared nerve injury surgery

The SNI model of chronic neuropathic pain included an axotomy and ligation of two of the three peripheral ramifications of the sciatic nerve, the tibial and common peroneal nerves and leaving the sural nerve intact, as described elsewhere (Decosterd and Woolf, 2000). The animals were lightly anesthetized with pentobarbital 0.5\% (Eutasil, Ceva Saúde Animal, Portugal). The common peroneal and tibial nerves were tightligated with 5.0 silk and sectioned distal to the ligation, removing 2$4 \mathrm{~mm}$ of the distal nerve stump. Great care was taken to avoid any contact with or stretching of the intact sural nerve. Muscle and skin were closed in two layers. Sham-controls involved exposure of the sciatic nerve and its branches without performing any manipulation.

\section{Nociceptive tests}

Nociceptive tests were performed in all animals a day before and two days after the surgery procedure, followed by testing every two days then forward, during the two months of experimental period. Both the ipsilateral (right hind paw) and the contralateral hind paw were tested in order to evaluate the presence of "mirror pain", described elsewhere as present in neuropathic pain pathologies (Tal and Bennett, 1994).

\section{Mechanical allodynia}

Animals were placed on an elevated wire grid and the lateral plantar surface of the paw stimulated with a series of ascending force von Frey monofilaments. The nociceptive threshold was taken as the lowest force that evoked a brisk withdrawal response to one of five repetitive stimuli (Tal and Bennett, 1994).

\section{Mechanical hyperalgesia}

With the animals on the elevated grid, a pin-prick test was performed using a safety pin. The lateral part of the plantar surface of the paw was briefly stimulated at intensity sufficient to touch but not penetrate the skin (Decosterd et al., 1998). The duration of paw withdrawal was measured, with an arbitrary minimal time of 0.5 seconds ( $\mathrm{s}$ ) (for the brief normal response) and maximal cut-off of $20 \mathrm{~s}$ (Tal and Bennett, 1994).
All behavioural tests were performed five days preceding animal 131 sacrifice during light period (9am to $6 \mathrm{pm}$ ) in a restricted group of 132 animals ( $n=18$ each group).

Anxiety-like behaviour - elevated plus-maze test (EPM)

Anxiety-like behaviour was evaluated in the EPM test through 135 an apparatus consisting of two open and two closed arms $(50.8 \times 136$ $10.2 \times 40.6 \mathrm{~cm}$ each arm) (MedAssociates Inc., St. Albans, Vermont, 137 USA). Each rat was placed in the centre of the elevated plus-maze 138 facing one of the open arms, and the time spent (s) in the open or 139 closed arms was recorded during a 5-min test period (Mesquita et al., 140 2006; Sousa et al., 2006). The elevated plus-maze was carefully 141 cleaned with $10 \%$ ethanol before each animal was placed on the 142 equipment.

Depressive-like behaviour - forced-swimming test (FST) 143 elsewhere (Porsolt et al., 1977, 1978). On day 1 (conditioning, pre- 146 test session), rats were individually placed in a clear Plexiglass 147 cylinder (29 $\mathrm{cm}$ in diameter and $50 \mathrm{~cm}$ in height) containing $30 \mathrm{~cm} 148$ of water $\left(25+0.5^{\circ} \mathrm{C}\right)$ and left to swim for $15 \mathrm{~min}$. The rats were then 149 removed from water and towel-dried, placed under a heating lamp 150

A
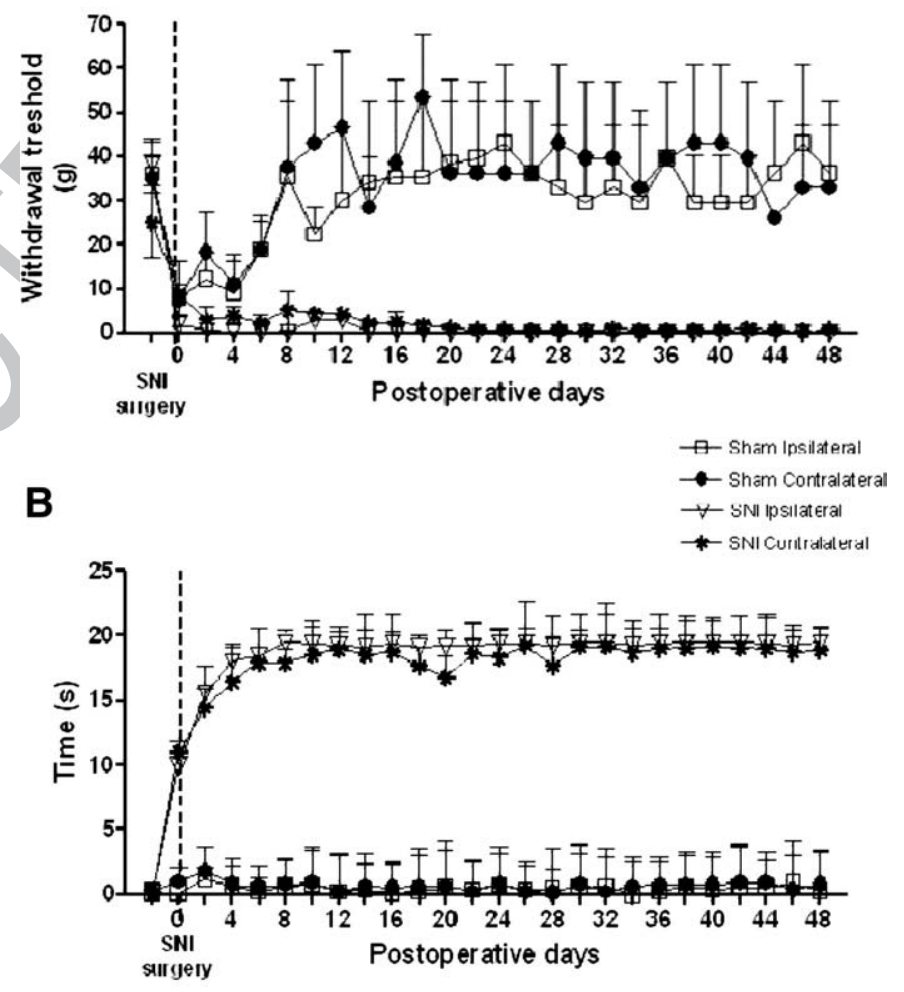

Fig. 1. Mechanical allodynia assessed by von Frey filaments (A) and mechanical hyperalgesia assessed by the pin-prick test (B) before and after surgery in SNI and Sham groups (dotted line indicates the day of SNI surgery). (A) Note that the presurgery threshold to von Frey filaments was similar in both SNI and Sham groups and in both hind paws; after surgery, the withdrawal threshold of the SNI group decreased within $24 \mathrm{~h}$ and remained low until the end of the 2 month experimental period. In Sham animals, the withdrawal threshold to von Frey filaments was decreased during the first postoperative days but returned to baseline values. (B) In the pin-prick test, SNI animals showed a strong hyperalgesia from the first postoperative day onwards, whereas Sham animals showed no hyperalgesia. The symbols and error bars represent mean +S.D. 
for $5 \mathrm{~min}_{\text {, }}$ and finally returned to their home cage. Twenty-four hours later, the rats were tested under the same conditions for 5 min (test session). Rats were judged to be immobile when both hind legs were not moving, and the rat was slightly bent forward (Mesquita et al., 2006).

Locomotion and exploratory behaviour - open field test (OF)

Motor activity and exploratory behaviour were evaluated by placing the rat into an infrared photobeam controlled open field activity test chamber in a brightly illuminated (white light) room. Animals were tested for $10 \mathrm{~min}$ in an arena $(43.2 \mathrm{~cm} \times 43.2 \mathrm{~cm}$ transparent acrylic walls and white floor) (MedAssociates Inc., St. Albans, Vermont) that was divided into a central and a peripheral zone. The time spent by each animal in the central and peripheral (residual) zone and its vertical activity (rearings) were the parameters evaluated in this test (Mesquita et al., 2006). Environmental odours were removed with $10 \%$ ethanol solution.

\section{Tissue preparation}

Both the SNI and Sham groups were divided as follows: i) in the first group ( $n=6$ each), designated to stereological analysis, the animals were anaesthetized with pentobarbital and perfused with $4 \%$ paraformaldehyde (PFA), the brains were removed, embedded in 2 hydroxyethyl glycol methacrylate, serially sectioned in a microtome at $30 \mu \mathrm{m}$ and stained with Giemsa; ii) in the second group ( $n=6$ each), designated to 3D-morphologycal analyses of dendritic arborization of AMY neurons, the animals were anesthetized with pentobarbital, perfused with saline and the brains were removed and processed for posterior staining following the Golgi-Cox method (Gibb and Kolb, 1998) and slicing in a vibratome at $100 \mu \mathrm{m}$; iii) in the third group ( $n=18$ each), processed for immunocytochemistry for detection of BrdU, GFAP (glial fibrillary acidic protein), NeuN (neuronal nuclei) and Calb (Calbindin), the animals were decapitated, the brains dissected, frozen in liquid nitrogen and sectioned in a cryostat $\left(-14^{\circ} \mathrm{C}\right)$.
Stereological procedures

183

The amygdaloid complex was subdivided in its nuclear compo- 184 nents as in Paxinos and Watson (1998): central (CeA), lateral (La), 185 basolateral anterior (BLA) and posterior (BLP), basomedial anterior 186 (BMA) and posterior (BMP) nuclei. The nuclei volume and cell number 187 estimation in AMY nuclei in every 8th section stained with Giemsa 188 was obtained through the Cavalieri's principle and optical fractionator 189 methods using the Stereoinvestigator software (MicroBrightField, Inc., 190 Williston, VT, USA).

3D-morphologycal analysis of dendrites

The brain sections stained with the Golgi-Cox method were 193 observed at the optical microscope and multipolar and bipolar AMY 194 neurons completely and perfectly stained (Cerqueira et al., 2007) were 195 considered for further analysis using the Neurolucida software 196 (MicroBrightField, Inc., Williston, VT, USA). The dendrites and spines 197 of 6 AMY neurons per animal were drawn.

All quantifications of markers for cell division and neuronal fate 200 were performed in the AMY. Positive controls for histochemical 201 reactions were confirmed by analysing the subgranular zone (SGZ) of 202 the hippocampus, since neuronal proliferation is known to occur in 203 this area (Gould et al., 1999a). As negative controls of immunocyto- 204 chemical reactions, the primary antibody was not included in the 205 protocol of each reaction; no specific immunoreaction was observed 206 following negative controls.

BrdU immunohistochemistry and quantification of BrdU-labelled cells 208

Bromodeoxyuridine (BrdU; an analogue of thymidine, incorpo- 209 rated into the newly synthesized DNA of replicating cells) incorpora- 210 tion was detected by immunocytochemistry on every 8th serial brain 211
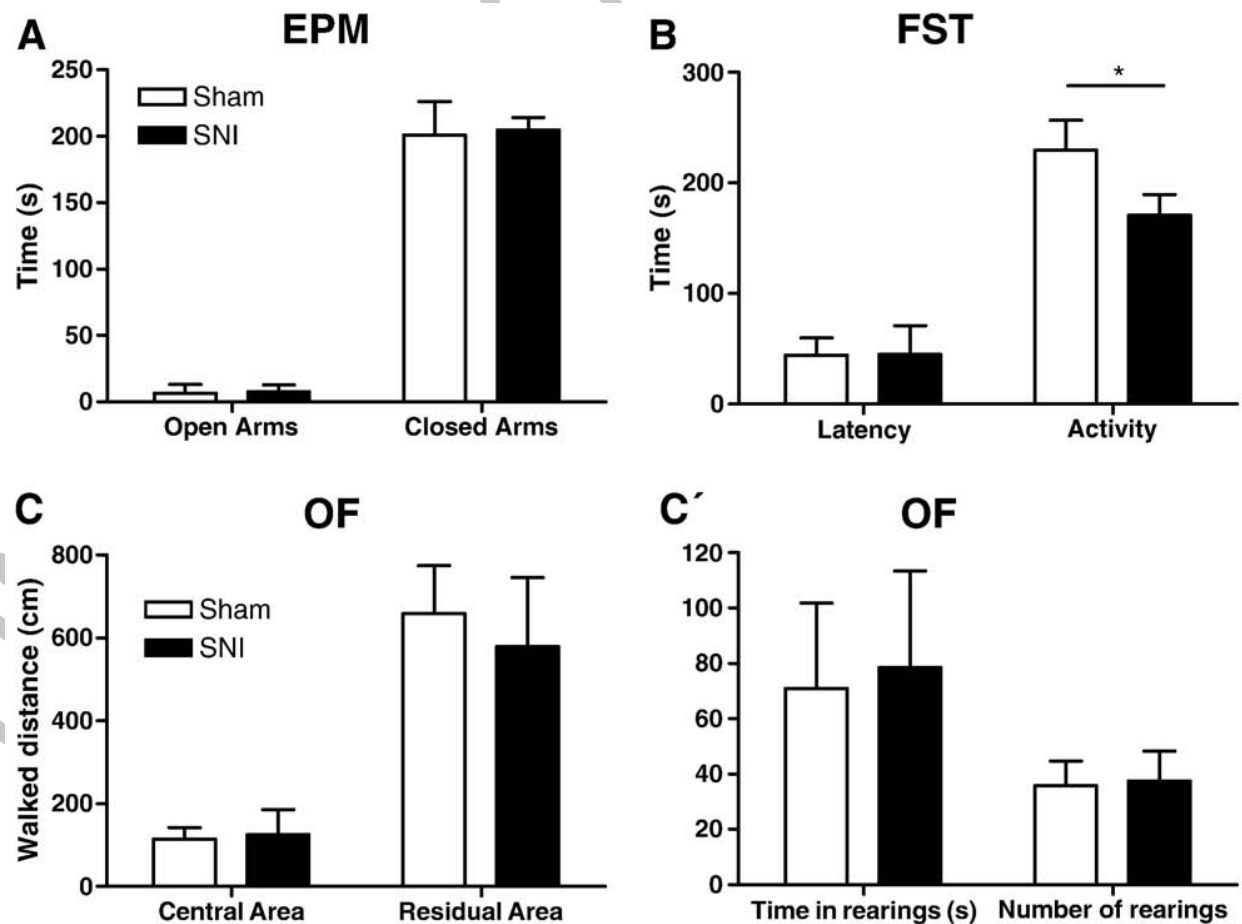

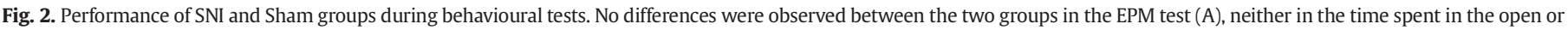

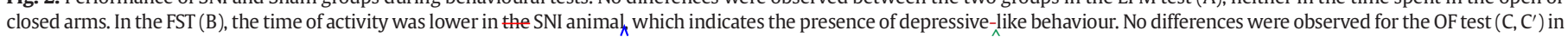
any of the parameters evaluated. 
section containing the amygdaloid complex. Briefly, sections were fixed in 4\% PFA for 30 minutes ( $\mathrm{min}$ ), permeabilized for $10 \mathrm{~min}$ in a solution containing $0.2 \%$ Triton $\mathrm{X}-100$ in Tris buffer saline (TBS) after a $3 \times 3 \mathrm{~min}$ wash in TBS, heated during $20 \mathrm{~min}$ in citrate buffer $0.1 \mathrm{M}$ following a $3 \times 3$ min wash and acidified in $\mathrm{HCl} 2 \mathrm{M}$ for 30 min after rinsing in distillated water. Endogenous peroxidase activity was blocked with $3 \% \mathrm{H} 2 \mathrm{O} 2$ in TBS for 10 min after a $3 \times 3$ min wash in TBS, followed by immersion in $4 \%$ bovine serum albumin (BSA) in TBS for $30 \mathrm{~min}$ (to block non-specific staining) after a $3 \times 3 \mathrm{~min}$ wash. After another $3 \times 3$ min wash in TBS, the tissue was incubated overnight with a primary monoclonal anti-BrdU antibody raised in mouse (1:50, Dako, Glostrup, DK) and stained cells were detected using a universal detection system (BioGenex, San Ramon, CA, USA) and diaminobenzidine (DAB $0.025 \%$ and $\mathrm{H} 2 \mathrm{O} 20.5 \%$ in Tris- $\mathrm{HCl} 0.05 \mathrm{M} \mathrm{pH} 7.2$ ), after a $3 \times 2$ min wash in TBS and a $1 \times 3$ min wash in Tris- $\mathrm{HCl}$, followed by counterstaining with haematoxylin. BrdU-positive cells were counted throughout the entire AMY area.

\section{Immunofluorescence and quantification of double-labelled cells}

Double-staining immunofluorescent reactions were performed in order to reveal three different groups: (i) BrdU and GFAP (glial fibrillary acidic protein; a marker of astrocyte glial cells; Reeves et al., 1989), (ii) BrdU and NeuN (protein expressed exclusively in mature neurons; Mullen et al., 1992) and (iii) BrdU and Calb (Calbindin; a calciumbinding protein present in functional mature neurons Meguro et al., 2004). The following primary antibody dilutions were used: rat antiBrdU (1:500, Accurrate, Westbury, MA), mouse anti-GFAP (1:500, Dako Glostrup, Denmark), mouse anti-NeuN (1:500, Chemicon International, Temecula, CA, USA) and rabbit anti-Calb (1:200, Chemicon International, Temecula, CA, USA). The initial protocol procedure (until the primary antibody incubation) was the same in the first three groups and similar to that described above for revealing BrdU. The following specific procedures for each double-staining method are explained briefly and separately for each group.

Brain sections were mounted in slides with Vectashield (Vector Laboratories, Burlingame, CA, USA) to delay fluorescence decay, and observed two days later in a fluorescence microscope. Data were confirmed posteriorly using confocal microscopy (Olympus FluoViewTM FV1000, OLYMPUS).

\section{i) BrdU and GFAP}

After overnight incubation with the primary antibody anti-BrdU raised in rat, sections were washed $3 \times 2 \mathrm{~min}$ in TBS and then incubated with a fluorescent Alexa 568 secondary antibody (goat anti-rat, 1:200; Molecular Probes, Eugene, OR) for ${ }_{\lambda} 1 h_{\lambda}$. Following a $3 \times 3$ min wash in TBS, sections were incubated during $3 h_{\lambda}$ with the primary antibody mouse anti-GFAP, followed by the fluorescent Alexa 488 secondary antibody (goat anti-mouse, 1:100, Molecular Probes, Eugene, $O R$ ) for $1 \mathrm{~h}$. The sections were finally washed $2 \times 2 \mathrm{~min}$ in TBS and $2 \mathrm{~min}$ in distillate water before being mounted in slides.

\section{ii) BrdU and NeuN}

Sections were incubated overnight with the primary antibody antiBrdU raised in rat followed by the fluorescent Alexa 568 secondary antibody (goat anti-rat, 1:200; Molecular Probes, Eugene, OR) for $1 h_{\lambda}$, after a $3 \times 3$ min wash in TBS. Then, sections were immersed for $3 \mathrm{~h}$ with the primary antibody anti-NeuN raised in mouse (1:500) and washed $3 \times 3 \mathrm{~min}$. Subsequently, they were incubated with biotinylated secondary antibody anti-mouse (1:200) for $1 \mathrm{~h}$ and, after a $3 \times 3$ min wash, incubated with Alexa Streptavidine 488 (1:100, Molecular Probes, Eugene, $\mathrm{OR}_{\mathcal{N}}$ for one final hour. The sections were washed in TBS and distillate water as above and mounted in slides.

\section{iii) BrdU and Calb}

Sections were incubated overnight with the rat anti-BrdU and mouse anti-Calb primary antibodies. In the next day, after a $3 \times 3$ min wash in TBS sections were firstly incubated with fluorescent Alexa 568274 (goat anti-mouse, 1:200) secondary antibody for $1 \mathrm{~h}$ and then with 275 fluorescent Alexa 488 (goat anti-rat, 1:200; Molecular Probes, Eugene, 276 OR) secondary antibody, after $\frac{\hat{A}}{\mathrm{a}} 3 \times 3 \mathrm{~min}$ wash. The sections were 277 washed in TBS and distillate water and mounted in slides.

Statistic analysis

For the analysis of baseline thresholds of SNI/Sham and ipsi/ 280 contralateral hind paws in the von Frey and pin-prick tests, one-way 281 analysis of variance (ANOVA) was performed. Considering that in the 282
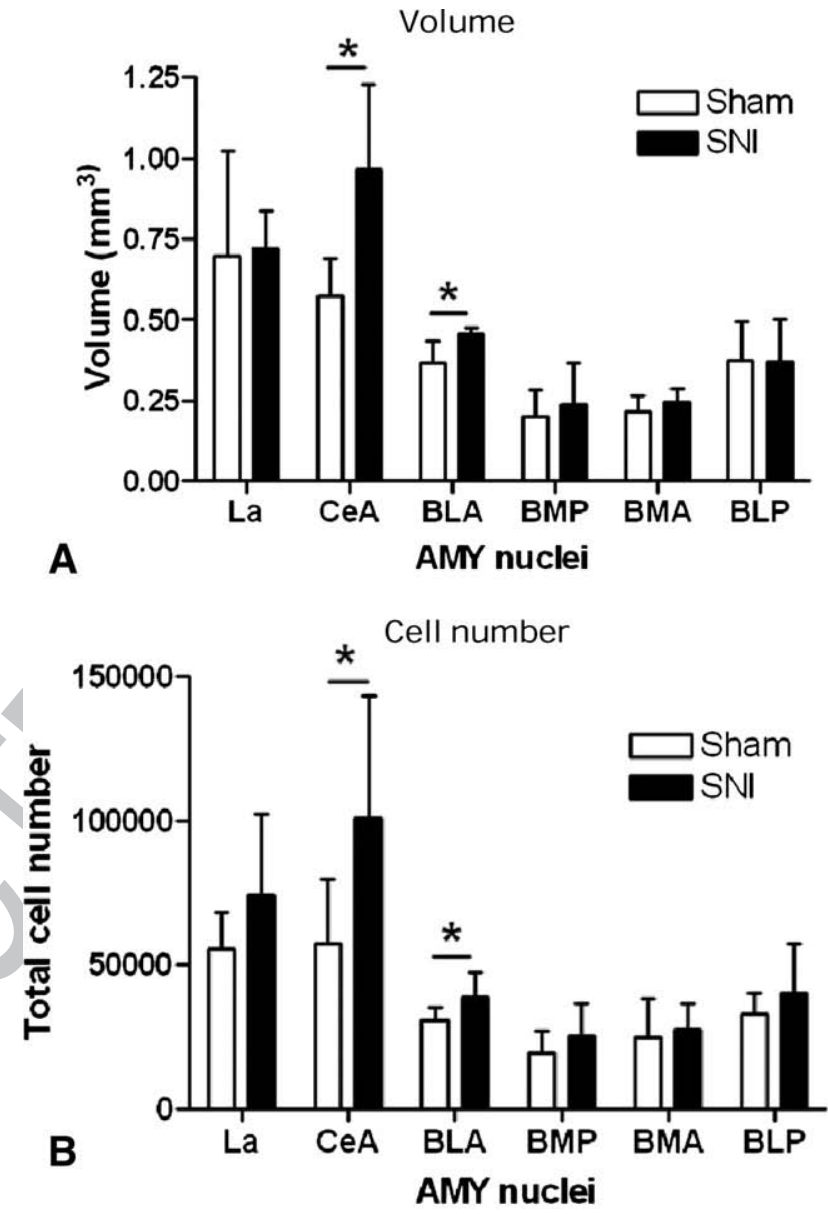

Total Dendritic Lenght

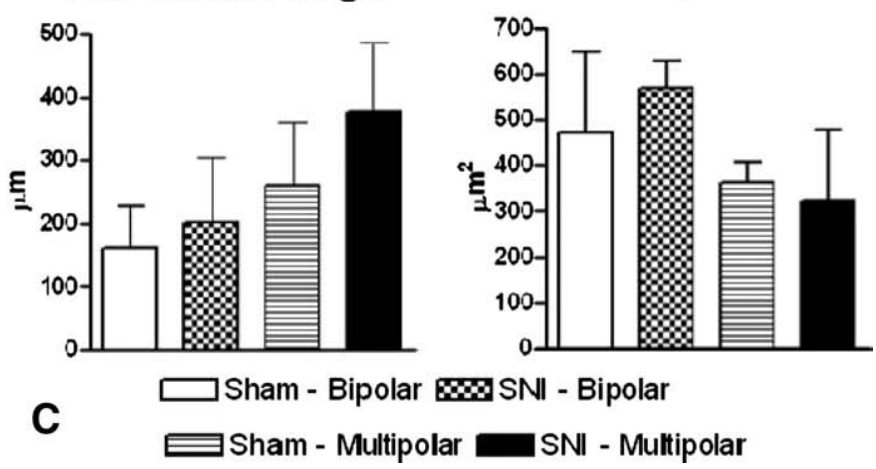

Fig. 3. Morphological analysis of AMY nuclei. (A) Volumes of different AMY nuclei were higher in neuropathic animals when compared to Sham, with differences being statistically significant for the CeA and BLA nuclei. (B) Cell number is also higher in all amygdalar nuclei of SNI animals, with differences being significant again in the CeA and BLA nuclei. (C) Structural analysis through Golgi-Cox method showed no differences in cell body volume and dendrite length of AMY neurons between SNI and Sham groups. 
rest of this study only comparisons between two groups were performed, the sStudent's t--test was used to analyse the results of all tests and procedures. The results were considered to be statistically different when $p<0.05$. Data are presented as mean \pm standard deviation ${ }_{\star}$

\section{Results}

The spared nerve injury model induces hypersensitivity for at least 2 months

Assessment of mechanical allodynia and hyperalgesia using, respectively, von Frey filament and pin-prick tests, were performed twice before the SNI surgery (baseline measurements) and every two days afterwards (during a two month period). Both neuropathic (SNI group) and sham-control (Sham group) animals presented a similar baseline withdrawal threshold measured by von Frey filaments (SNI: ipsilateral $38 \pm 6.1 \mathrm{~s}$ contralateral $25 \pm 8.2 \mathrm{~s}$. Sham: ipsilateral $36 \pm 7.3 \mathrm{~s}$, contralateral $35 \pm 5.1_{\kappa}$ Fig. $1 \mathrm{~A}$ ). A bilateral decrease in nociceptive threshold was observed in neuropathic animals within $24 \mathrm{~h}$ after surgery. This threshold decrease reached the level of 0-5 g five days after the surgery, a value that remained constant until the end of the two month experimental period. These data showed that the SNI group developed and maintained a strong mechanical allodynia in both hind paws, as a consequence of the surgery. On the contrary, nociceptive 304 threshold in Sham animals decreased slightly with the sham surgery, 305 returning to baseline values within a week, never reaching thresholds 306 as low as those presented by SNI animals (Fig. 1A). In what concerns the 307 pin-prick test, the baseline duration of hyperalgesic behaviour was less 308 than $1 \mathrm{~s}$ in all animals, and there were no differences between groups 309 (SNI: ipsilateral $0.17 \pm 0.17 \mathrm{~s}$, contralateral $0.11 \pm 0.8 \mathrm{~s}$; Sham: ipsilateral 310 $0.13 \pm 1.11 \mathrm{~s}$, contralateral $0.2 \pm 0.2$; Fig. 1B). Within $24 \mathrm{~h}$ from the 311 surgery, SNI animals reached the maximal duration of hyperalgesic 312 behaviour in both hind paws (20 s) whereas no changes were observed 313 in Sham animals (Fig. 1B). These data showed that the SNI group 314 developed and maintained a clear hyperalgesic state during virtually 315 the entire experimental period. In summary, data on pain-related 316 behaviour demonstrated that SNI animals developed a clear neuro- 317 pathy that extended throughout the complete experimental period. 318

Neuropathic animals develop a depressive-like behaviour but do not 319 display signs of increased anxiety

Emotional behaviour was assessed seven weeks after the surgery. 321 EPM was performed to evaluate anxious behaviour, FST to assess 322 depressive-like behaviour and the OF test to determine locomotion 323 and exploratory behaviour (Mesquita et al., 2006). In the EPM, no 324 differences were found in the behavioural responses between SNI and 325

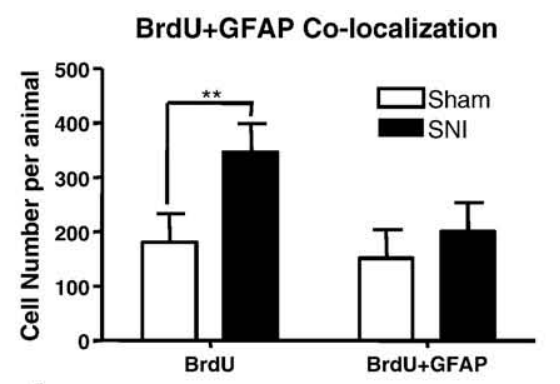

A
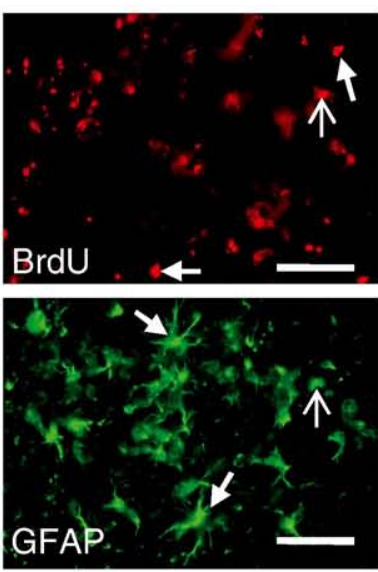

A

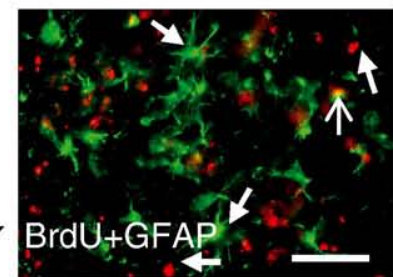

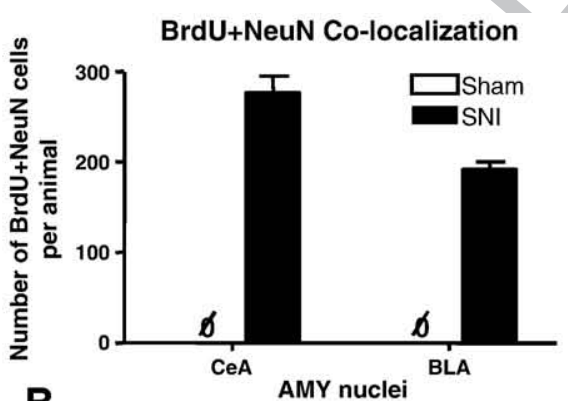

B
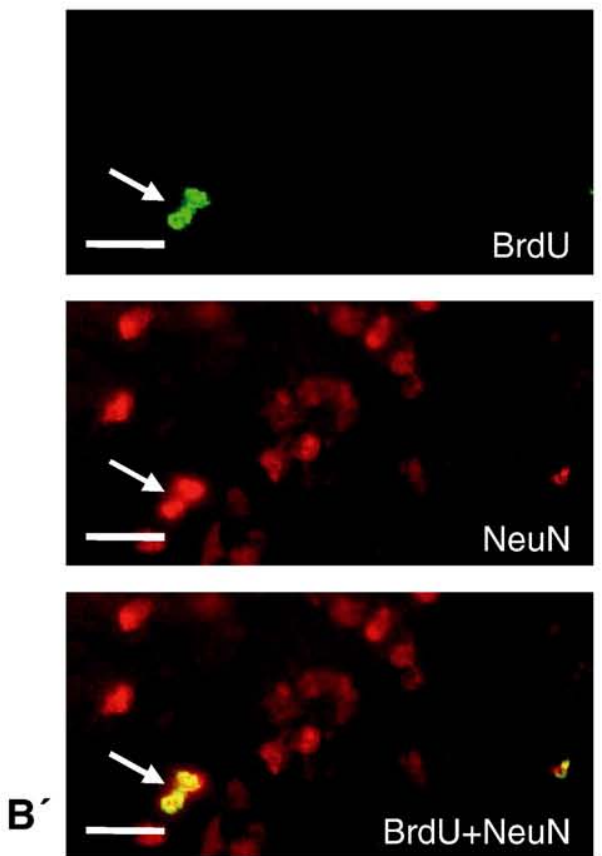

BrdU+Calb Co-localization

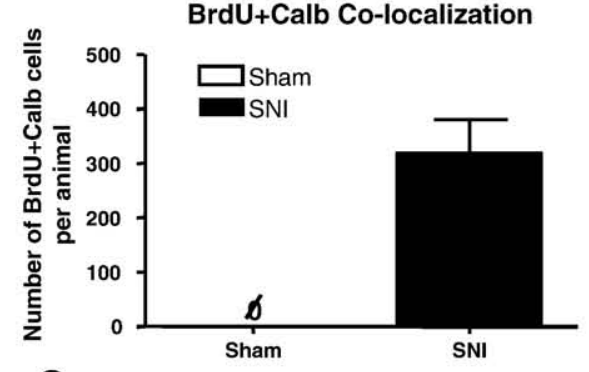

C
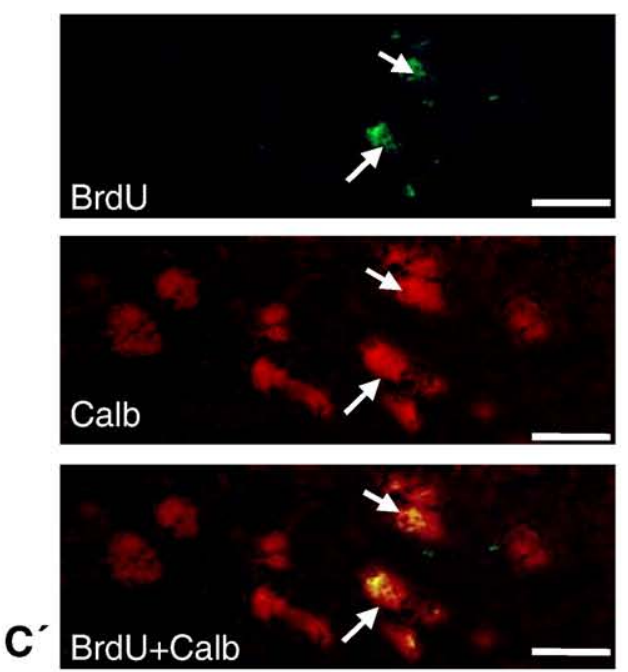

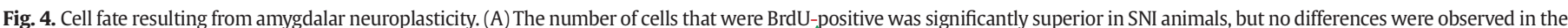

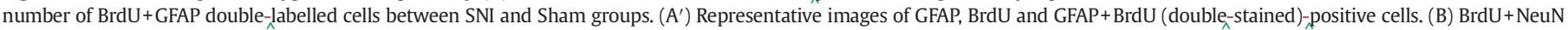

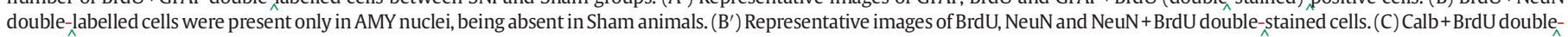
stained cells were also present only in neuropathic animals. $\left(C^{\prime}\right)$ Representative images of BrdU, Calb and Calb+BrdU double-stained cells. Magnification bar: $60 \mu \mathrm{m}\left(\mathrm{A}^{\prime}\right), 20 \mu \mathrm{m}\left(\mathrm{B}^{\prime}, \mathrm{C}^{\prime}\right)$. 
Sham groups (Fig. 2A), thereby showing that the anxiety levels were unaltered by induction of SNI. On the other hand, the FST revealed significant differences between experimental groups (Fig. 2B): while Sham animals were active for $230 \pm 27 \mathrm{~s}$, SNI animals only tried to escape/swim for $180 \pm 38 \mathrm{~s}(p=0.012)$, which indicates the presence of a learned helplessness (depressive-like) behaviour in neuropathic animals. Since FST test includes movement of the paws and neuropathic animals are hyperalgesic and allodynic in both ipsilateral and contralateral hind paws, the OF test was performed in order to validate the FST test. This test revealed that the SNI group had no differences in the locomotion ability when compared with Sham group and it also revealed that the number of rearings (an indicator of exploratory behaviour) did not differ between experimental groups (Figs. 2C, $\mathrm{C}^{\prime}$ ). The absence of differences in the time spent in central vs. peripheral part of the OF arena also indicates the absence of altered anxiety behaviour in neuropathic animals. In summary, these behavioural studies demonstrate that a 2 month neuropathy induced a depressive-like, but not anxious-like, behaviour.

\section{Volume and cell number are increased in amygdaloid nuclei}

After animal perfusion, 6 brains of each experimental group were prepared for stereological analysis and other 6 SNI and Sham brains were processed for tri-dimensional morphological analysis. For stereological analysis the AMY was divided in 6 nuclei (Paxinos and Watson, 1998): central (CeA), lateral (La), basolateral anterior (BLA) and posterior (BLP), basomedial anterior (BMA) and posterior (BMP).
We found a general increase in the volume of all these nuclei in SNI 351 neuropathic animals, with a significant increase being observed in CeA 352 $(p=0.02)$ and BLA $(p=0.019)$ nuclei (Fig. 3A). In order to determine the 353 causes for these structural changes of AMY, we analysed potential 354 alterations in cell numbers and cellular volumes. SNI neuropathic 355 animals showed a general increase in the number of cells in all AMY 356 nuclei, with a significant difference being present again in CeA 357 $(p=0.015)$ and BLA $(p=0.016)$ nuclei (Fig. 3B). On the contrary, 3D- 358 morphological analysis revealed no significant differences in dendritic 359 lengths (Fig. 3C) or perikarya areas (Fig. 3D) between neuropathic and 360 Sham animals, both in bipolar and multipolar AMY neurons. Taken 361 together, these results indicate that the significant increase observed 362 in CeA and BLA nuclear volumes of SNI animals was due, at least in 363 part, to an increase in cell numbers.

Newborn neurons contribute to increased cell numbers in AMY

365

Rats received one injection of the cell proliferation marker 366 bromodeoxyuridine (BrdU) in the three consecutive days before 367 their sacrifice. The aim of this procedure was to determine if cell 368 proliferation was responsible for the higher number of cells observed 369 in the CeA and BLA nuclei in SNI animals. Immunohistochemistry 370 revealed the presence of BrdU-positive cells in the AMY of both 371 SNI and Sham groups, but with significantly higher numbers in 372 neuropathic animals $(p=0.001$; Fig. $4 \mathrm{~A})$. In order to identify the 373 phenotype of these newly-acquired cells, two different double- 374 staining immunohistochemistry reactions were performed: BrdU +375
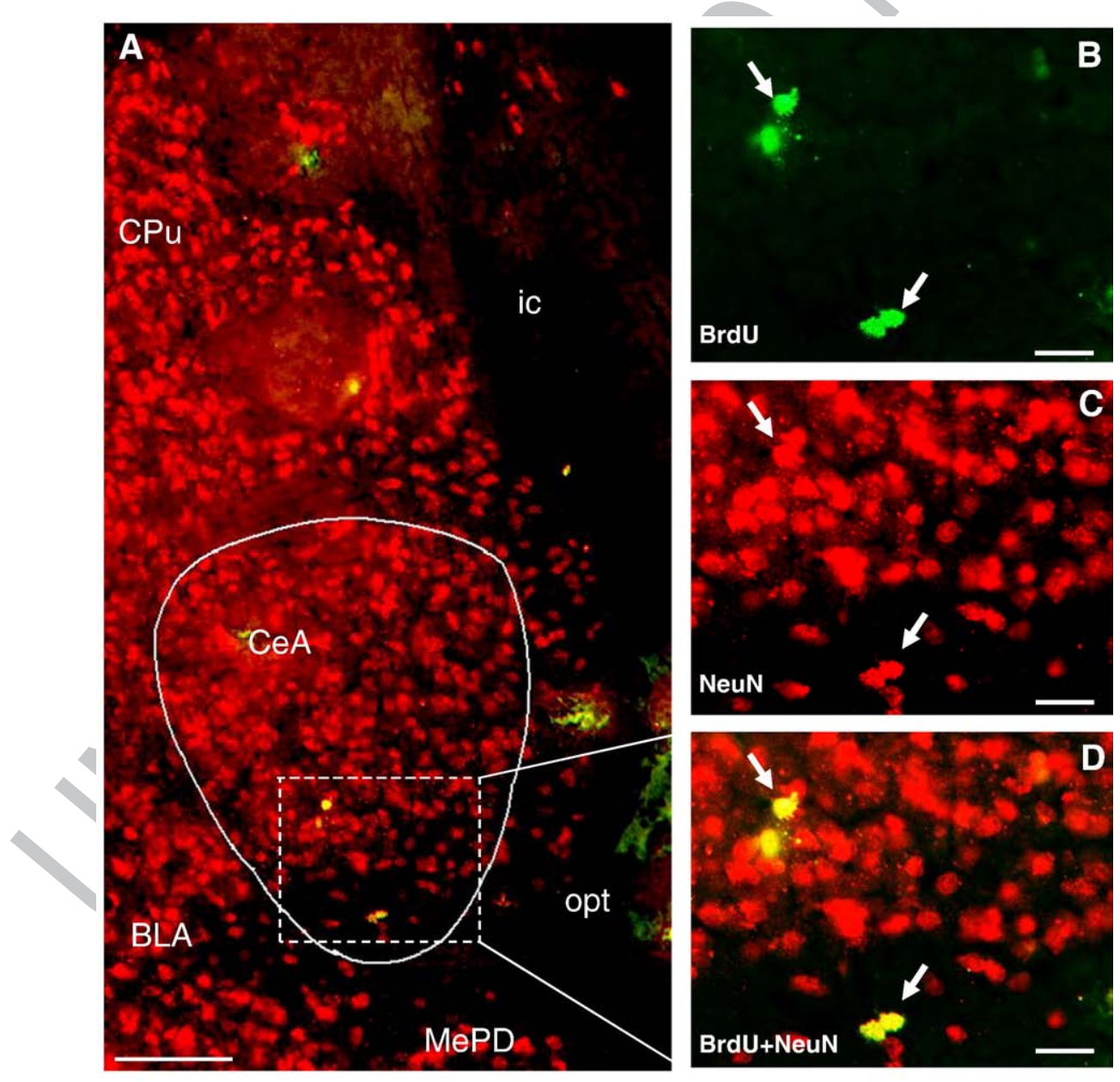

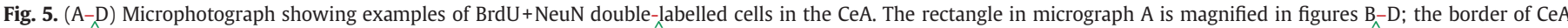

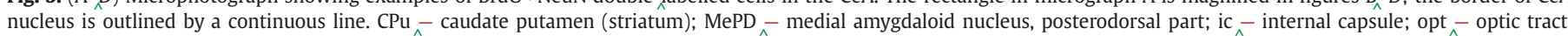
Magnification bar: $100 \mu \mathrm{m}(\mathrm{A}), 20 \mu \mathrm{m}(\mathrm{B}-\mathrm{F})$. 

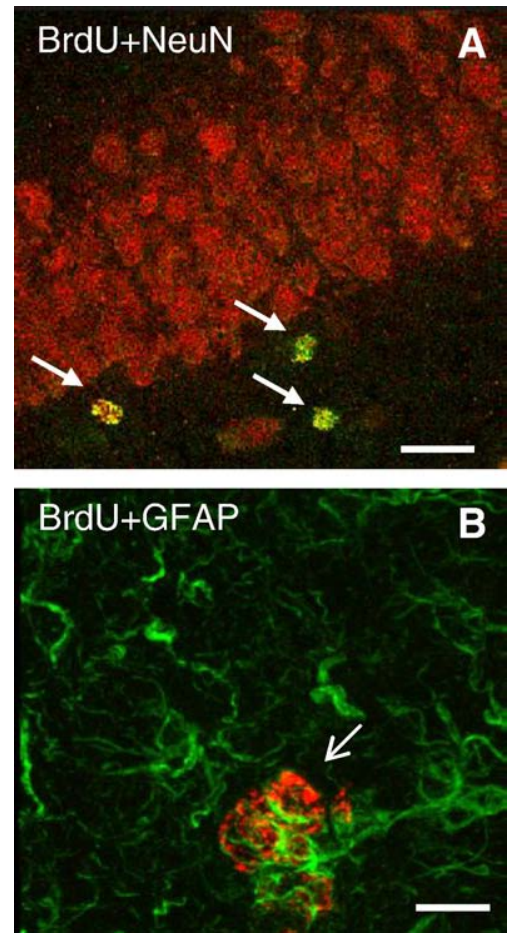

Fig. 6. Examples of BrdU +NeuN (A) and BrdU +GFAP (B) double-labelled cells (arrows) obtained in positive-control sections from the subgranular zone of the dentate gyrus of the hippocampus. Magnification bar: $20 \mu \mathrm{m}$ (A), $10 \mu \mathrm{m}$ (B).

glial fibrillary acidic protein marker (GFAP) and BrdU + post-mitotic neuronal marker (NeuN). The number of BrdU +GFAP-positive cells was similar between the SNI and Sham groups. On the other hand, BrdU + NeuN double-labelled cells were observed only in the SNI group; interestingly, they were mainly located in the CeA and BLA nuclei (Figs. 4B, $B^{\prime}, 5$ ). These findings indicate the presence of newly proliferating neurons in the AMY after prolonged SNI, as further demonstrated by the presence of BrdU + Calbindin-positive cells in the AMY of neuropathic animals (Fig. $4 \mathrm{C}, \mathrm{C}^{\prime}$ ). Positive control sections obtained from the subgranular zone of the hippocampal dentate gyrus showed the presence of both BrdU + NeuN and BrdU + GFAP double-labelled cells (Fig. 6).

In summary, data demonstrate not only that recently-divided newborn neurons are formed in the AMY of chronic pain animals, but also that these neurons reach a physiologically mature (i.e., functional) state.

\section{Discussion}

After two months of neuropathic pain, SNI animals exhibited signs of sustained persistent pain associated with a significant depressive-like behaviour. At the CNS level, a structural reorganization of the amygdaloid complex was observed that was associated with a significant increase in the volume of the basolateral (BLA) and central (CeA) AMY nuclei. The volume increase was due to an increased number of AMY cells, and not to hypertrophy of dendrites or perikarya of amygdalar neurons. The present study is the first demonstrating cell proliferation in a limbic area, as a result of chronic neuropathic pain. Earlier, only electrophysiological studies have shown chronic pain-related neuroplasticity of AMY neurons in persistent arthritis, visceral pain (Han and Neugebauer, 2004) or neuropathy (Ikeda et al., 2007). Moreover, this is the first study demonstrating that chronic pain results in depressive-like behaviour associated with neuroplasticity in a major brain centre implicated in the control of both emotions and pain.
Changes in emotional behaviour and neuroplasticity in the AMY

Morphological plasticity in the AMY was previously suggested in 410 cases of prolonged emotional disturbance, as shown by increased AMY 411 volumes measured by structural magnetic resonance in patients with 412 depression and anxiety (Frodl et al., 2002; Tebartz van Elst et al., 2000). 413 Clinical data also reveal that prolonged pain conditions are associated 414 with a high incidence of emotional disorders, including anxiety and 415 depression (Rasmussen et al., 2004). Herein, we show that in the rat, a 416 two month neuropathy resulted also in a depressive-like behaviour 417 measured by the forced-swimming test (FST), but no alterations in 418 anxiety levels were detected in the elevated plus-maze and open field 419 tests. We also show that increased immobility time in the FST should 420 not be ascribed to motor impairments as there were no changes in 421 locomotor activity and exploratory behavior. As in humans, SNI 422 neuropathy associated with emotional alterations may result from, 423 or contribute to, the structural changes observed in the AMY. It has 424 been proposed that the increase in AMY volume observed in de- 425 pressive patients was a consequence of the continuous prolonged 426 activation of this area (Frodl et al., 2002). Following the same rationale, 427 the present increase in AMY volume may result from the continuous 428 flow of nociceptive information into AMY regions receiving sensory 429 information (including the BLA) and the consequent prolonged activity 430 of AMY neurons triggering the appropriate response action (CeA is the 431 main effector of AMY). Especially relevant is the increase in the CeA 432 volume, as its latero-capsular part is defined as the 'nociceptive 433 amygdala' due to its high content in neurons implicated in nociceptive 434 processing (Bernard et al., 1996; Neugebauer and Li, 1992; Neugebauer 435 et al., 2004).

The volume increase in the AMY after two months of neuropathic 437 pain may have resulted from one or various different processes: cell 438 size (soma and dendritic size) increase, cell number (neurons or glial 439 cells) increase, or increased extracellular volume. However, subse- 440 quent analysis revealed that the increased volume of the AMY in SNI 441 animals could not be ascribed to cell size variations, but rather to an 442 increase in cell number. Interestingly, such increase in cell numbers 443 was confirmed by the observation of newly proliferating cells in AMY 444 nuclei of SNI animals. Although the presence of newborn neurons in 445 the adult brain of mammals is considered to be restricted to two areas, 446 the subgranular zone (SGZ) of the hippocampus and the subventricular 447 zone (SVZ) (Doetsch et al., 1997; Gould et al., 1999b; Kempermann and 448 Gage, 2000), the possibility of neurogenesis in the AMY has already 449 been raised in a study showing evidence for the presence of newly 450 generated neurons in the AMY of adult primates, at basal conditions 451 (Bernier et al., 2002). The results of double-immunoreactions (BrdU+ 452 NeuN) performed in the present study demonstrate that a significant 453 number of these newly-born cells undergo a neuronal phenotype. 454 Thus, the genesis of newborn neurons is responsible, at least in part, 455 for the increase in cell number underlying the increase of volume 456 observed in the AMY of SNI animals. In contrast, the number of cells 457 stained simultaneously for markers of cell proliferation (BrdU) and glia 458 (GFAP) revealed no additional glial cell proliferation in the AMY 459 following SNI induction; this indicates that SNI results only in ad- 460 ditional neuronal proliferation, with a similar basal rate of astrocyte 461 cell division being common to both Sham and SNI animals.

Our observation of NeuN and BrdU co-localization in AMY cells 464 indicate that newly generated cells reached neuronal maturation in the 465 amygdaloid complex. This is in accordance with the time points for 466 expression of neuronal differentiation markers described by Kemper- 467 mann et al. (2004) and Steiner et al. (2004): in the hippocampus of adult 468 mice NeuN expression becomes higher than immature-neuron 469 markers 3 days after cell division. Additionally, the presence of BrdU+ 470 Calb double-labelled neurons in the AMY confirms the maturation and 471 
phenotypical differentiation of newborn neurons in definitive AMY of SNI animals.

Whether these newly-born cells observed in the AMY of SNI rats result from local progenitor cells or migrate from adjacent neurogenic regions is still not known. However, several studies have shown that besides the normal migration of proliferative cells from the SVZ to the olfactory bulb (through the rostral migratory stream, RMS) or from the SGZ to other areas of the DG, they can migrate from the SVZ to injured areas of the brain (Iwai et al., 2003; Parent et al., 2002; Van Kampen et al., 2004). Therefore, it is possible that the new neurons here observed have their origin in SVZ progenitor cells that, through migration, reached the amygdaloid complex following the prolonged pain syndrome induced by the SNI model. Supporting this hypothesis, post-natal neurogenesis in the SVZ and SGZ can be regulated positively through the enhancement of the survival of newly generated cells and negatively through the down regulation of cell proliferation (Gould and Gross, 2002) following different stimuli (Jin et al., 2001). On the other hand, a growing amount of evidence supports the notion that the CNS itself is not as static as once believed: BrdU-positive cells were shown to be present in several regions of the adult CNS currently thought to be mitotically quiescent (Rietze et al., 2000); studies report that neurogenesis is prone to occur in other areas of adult mammals, like the neocortex (Gould et al., 1999a; Takemura, 2005), the striatum (Van Kampen et al., 2004; Bedard et al., 2006), the substantia nigra (Yoshimi et al., 2005) and the amygdala itself (Bernier et al., 2002). Taking into account these data, it should not be excluded the possibility that neural stem cells could be present in the AMY and proliferate following the prolonged neuropathy resulting from the SNI model. Further experimental procedures must be performed to elucidate this issue.

\section{Roles of AMY in pain and emotional processing}

Several data implicate the AMY in pain modulation, as shown by changes in pain tolerance induced by AMY manipulation (Manning, 1998). Moreover, the AMY has a role in both pain inhibition and pain facilitation (Manning and Mayer, 1995; Manning et al., 2001; Tershner and Helmstetter, 2000). This dual effect may result from direct AMY projections to brainstem areas implicated in both descending antinociception and pronociception (Almeida et al., 1999; Bouhassira et al., 1992; Porreca et al., 2002). As a balance between descending inhibiting (antinociceptive) and facilitating (pronociceptive) actions upon spinal nociceptive transmission can contribute to the normal control of pain perception (Lima and Almeida, 2002; Pertovaara, 2000; Porreca et al., 2002; Ren and Dubner, 1996; Schaible et al., 1991), the AMY may have a crucial role as a higher centre modulating the brainstem pain centres responsible for the fine regulation of the spinal nociceptive transmission. Thus, it is possible that the here observed amygdalar neuroplasticity may contribute not only to emotional changes but also to alterations in nociception. In support of this hypothesis, volume changes of AMY were already shown in imaging studies of patients with a major depression (Drevets, 2000) and changes in synaptic function of nociceptive AMY neurons have been described in sustained pain conditions (Han and Neugebauer, 2004; Ikeda et al., 2007). Additionally, the neuronal proliferation observed in AMY areas involved in afferent (BLA) and efferent (CeA) nociceptive processing may disrupt fine neuronal networks between high brain centres, which provide a structural basis for deregulation of emotional behaviour.

\section{Conclusion}

In conclusion, this study shows that besides mechanical hyperalgesia and allodynia, animals subjected to the SNI model of neuropathic pain during a two month period developed a depressive-like behaviour associated with an increased volume of AMY nuclei that results from cell proliferation. Importantly, this is the first study providing evidence for 534 the presence of newly-born cells in the amygdaloid complex as a 535 consequence of a sustained chronic (neuropathic) pain condition. We 536 hypothesize that these neuroplastic changes of the AMY could be 537 associated with the development of depressive-like behaviour in 538 neuropathic animals. Nonetheless, future studies on the origin of 539 newborn neurons and their integration in the pre-existing synaptic 540 network should be performed in order to determine the relevance of this 541 phenomenon.

\section{Uncited-references}

Aliashkevich et al., 2003

Almeida et al., 2002

Coutaux et al., 2005

Gage, 2002

Gageet al., 1998

Kuhn et al., 1996

Kumar et al., 2005

LeDoux et al., 1990

McEwen, 2003

McQuay et al., 1996

Miniet al., 1995

Reynolds and Weiss, 1992

Shimet al., 2005

Faub, 1982

Vanegas and Schaible, 2004

Watkins and Maier, 2002

Weiss et al., 1996

Zhaoet al., 2003

545

546

This study was supported by Fundação para a Ciência e Tecnologia 563 (FCT) Project no. POCTI/NSE/46399/2002, FEDER and Fundação 564 Calouste Gulbenkian Project no. 74551. Leonor Gonçalves is a PhD 565 Fellow supported by FCT.

Aliashkevich, A.F., Yilmazer-Hanke, D., Van Roost, D., Mundhenk, B., Schramm, J., 568 Blumcke, I., 2003. Cellular pathology of amygdala neurons in human temporal lobe 569 epilepsy. Acta Neuropathol. (Berl) 106, 99-106.

Almeida, A., Størkson, R., Lima, D., Hole, K., Tjølsen, A., 1999. The medullary dorsal 571 reticular nucleus facilitates pain behaviour induced by formalin in the rat. Eur. J. 572 Neurosci. 11, 110-122.

Almeida, A., Cobos, A., Tavares, I., Lima, D., 2002. Brain afferents to the medullary dorsal 574 reticular nucleus: a retrograde and anterograde tracing study in the rat. Eur. J. 575 Neurosci. 16, 81-95.

Almeida, A., Leite-Almeida, H., Tavares, I., 2006. Medullary control of nociceptive 577 transmission: reciprocal dual communication with the spinal cord drug discovery 578 today: disease mechanisms, 3, pp. 305-312.

Altshuler L Bookheimer S, Proenza, M. A. Townsend J Sabb, F, Firestine, A Bartzokis, 580 G., Mintz, J., Mazziotta, J., Cohen, M.S., 2005. Increased amygdala activation during 581 mania: a functional magnetic resonance magnetic resonance imaging study. Am. J. 582 Psychiatry 162, 1211-1213.

Anand, KJ. Craig, K.D. 1996. New perspectives on the definition of pain. Pain 70,209-211. 58 Bedard, A., Gravel, C., Parent, A., 2006. Chemical characterization of newly generated 585 neurons in the striatum of adult primates. Exp. Brain Res. 170, 501-512. 586

Bernard, J.F. Bester, H., Besson, J.M. 1996. Involvement of the spino-parabrachio- 587 amygdaloid and -hypothalamic pathways in the autonomic and affective emotional 588 aspects of pain. Prog. Brain Res. 107, 243-255.

Bernier, P.J., Bedard, A., Vinet, J., Levesque, M., Parent, A., 2002. Newly generated 590 neurons in the amygdala and adjoining cortex of adult primates. Proc. Natl. Acad. 591 Sci. U. S. A. 99, 11464-11469.

Bouhassira, D., Villanueva, L., Le Bars, D., 1992. Effects of systemic morphine on diffuse 593 noxious inhibitory controls: role of the periaqueductal grey. Eur. J. Pharmacol. 216, 594 149-156.

Bremner, J.D., Narayan, M., Anderson, E.R., Eric, R., Staib, L.H., Miller, H.L., Charney, D.S., 596 2000. Hippocampal volume reduction in major depression. Am. J. Psychiatry 157, 597 $115-117$.

Cerqueira, J.J., Taipa, R., Uylings, H.B., Almeida, O.F., Sousa, N., 2007. Specific configuration of 599 dendritic degeneration in pyramidal neurons of the medial prefrontal cortex induced 600 by differing corticosteroid regimens. Cereb. Cortex 17, 1998-2006. 
Coutaux, A., Adam, F., Willer, J.C., Le Bars, D., 2005. Hyperalgesia and allodynia: peripheral mechanisms. Jt. bone spine 72, 359-371.

Danziger, N., Weil-Fugazza, J., Le Bars, D., Bouhassira, D., 2001. Stage-dependent changes in the modulation of spinal nociceptive neuronal activity during the course of inflammation. Eur. J. Neurosci. 13, 230-240.

Davis, M., Whalen, P.J., 2001. The amygdala: vigilance and emotion. Mol. Psychiatry 6, $13-34$.

Decosterd, I., Woolf, C.J., 2000. Spared nerve injury: an animal model of persistent peripheral neuropathic pain. Pain 87, 149-158.

Decosterd, I., Buchser, E., Gilliard, N., Saydoff, J., Zurn, A.D., Aebischer, P., 1998 Intrathecal implants of bovine chromaffin cells alleviate mechanical allodynia in a rat model of neuropathic pain. Pain 76, 159-166.

Devor, M., 2006. Sodium channels and mechanisms of neuropathic pain. J. Pain 7 (Suppl 1), S3-S12 Review.

Doetsch, F., Garcia-Verdugo, J.M., Alvarez-Buylla, A., 1997. Cellular composition and three-dimensional organization of the subventricular germinal zone in the adult mammalian brain. J. Neurosci. 17, 5046-5061.

Drevets, W.C., 2000. Neuroimaging studies of mood disorders. Biol. Psychiatry 48, $813-829$.

Frodl, T., Meisenzahl, E., Zetzsche, T., Bottlender, R., Born, C., Groll, C., Jäger, M. Leinsinger, G., Hahn, K., Möller, H.-J., 2002. Enlargement of the amygdala in patients with a first episode of major depression. Biol. Psychiatry 51, 708-714.

Gao, X., Kim, H.K., Chung, J.M., Chung, K., 2005. Enhancement of NMDA receptor phosphorylation of the spinal dorsal horn and nucleus gracilis neurons in neuropathic rats. Pain 116, 62-72.

Gage, F.H., 2002. Neurogenesis in the adult brain. J. Neurosci. 22, 612-613.

Gage, F.H., Kempermann, G., Palmer, T.D., Peterson, D.A., Ray, J., 1998. Multipotent progenitor cells in the adult dentate gyrus. J. Neurobiol. 36, 294-266.

Gibb, R., Kolb, B., 1998. A method for vibratome sectioning of Golgi-Cox stained whole rat brain. J. Neurosci. Methods 79, 1-4.

Gonçalves, L., Silva, R., Pinto-Ribeiro, F., Pego, J.M., Bessa, J.M., Pertovaara, A., Sousa, N., Almeida, A., 2006. Chronic neuropathic pain induces neurogenesis in the rat amygdala and is associated with altered emotional behavior. Society Neurosci Abstr, No 443.17, Abstract Viewer/Itinerary Planner.

Gould, E., Gross, C.G., 2002. Neurogenesis in adult mammals: some progress and problems. J. Neurosci. 22, 619-623.

Gould, E., Reeves, A.J., Graziano, M.S., Gross, C.G., 1999a. Neurogenesis in the neocortex of adult primates. Science 286, 548-552.

Gould, E., Reeves, A.J., Fallah, M., Tanapat, P., Gross, C.G., 1999b. Hippocampal neurogenesis in adult Old World primates. Proc. Natl. Acad. Sci. U. S. A. 96, 5263-5267.

Han, J.S., Neugebauer, V., 2004. Synaptic plasticity in the amygdala in a visceral pain model in rats. Neurosci. Lett. 361, 254-257.

kkeda, R., Takahashi, Y., Inoue, K., Kato, F., 2007. NMDA receptor-independent synaptic plasticity in the central amygdala in the rat model of neuropathic pain. Pain 127, $161-172$.

wai, M., Sato, K., Kamada, H., Omori, N., Nagano, I., Shoji, M., Abe, K., 2003. Temporal profile of stem cell division, migration, and differentiation from subventricular zone to the olfactory bulb after transient forebrain ischemia in gerbils. J. Cereb. Blood Flow Metab. 23, 331-341.

Jin, K., Minami, M., Lan, J.O., Mao, X.O, Batteur, S, Simon, R.P., Greenberg, D.A, 2001. Neurogenesis in the dentate subgranular zone and rostral subventricular zone after focal cerebral ischemia in the rat. Proc. Natl. Acad. Sci. U. S. A. 98, 4710-4715.

Kauppila, T., Xu, X.J., Yu, W., Wiesenfeld-Hallin, Z., 1998. Dextromethorphan potentiates the effect of morphine in rats with peripheral neuropathy. Neuroreport 9, 1071-1074.

Kempermann, G., Gage, F.H., 2000. Neurogenesis in the adult hippocampus. Novartis Found. Symp. 231, 220-235 discussion 235-241, 302-306.

Kempermann, G., Jessberger, S., Steiner, B., Kronenberger, G., 2004. Milestones of neuronal development in the adult hippocampus. Trends Neurosci, 27, 447-552.

Kuhn, H.G., Dickinson, A.H., Gage, F.H., 1996. Neurogenesis in the dentate gyrus of the adult rat: age-related decrease of neuronal progenitor proliferation. J. Neurosci. 16 2027-2033.

Kumar, A.M., Solano, M.P., Fernandez, J.B., Kumar, M., 2005. Adrenocortical response to ovine corticotropin-releasing hormone in young men: cortisol measurement in matched samples of saliva and plasma. Horm. Res. 64, 55-60.

LeDoux, J.E., Cicchetti, P., Xagoraris, A., Romanski, L.M., 1990. The lateral amygdaloid nucleus: sensory interface of the amygdala in fear conditioning. J. Neurosci. 10, 1062-1069.

Lima, D., Almeida, A., 2002. The medullary dorsal reticular nucleus as a pronociceptive centre of the pain control system. Prog. Neurobiol. 66, 81-108.

Manning, B.H., 1998. A lateralized deficit in morphine antinociception after unilateral inactivation of the central amygdala. J. Neurosci. 18, 9453-9470.

Manning, B.H., Mayer, D.J., 1995. The central nucleus of the amygdala contributes to the production of morphine antinociception in the rat tail-flick test. J. Neurosci. 15 , 8199-8213.

Manning, B.H., Merin, N.M., Meng, I.D., Amaral, D.G., 2001. Reduction in opioid- and cannabinoid-induced antinociception in rhesus monkeys after bilateral lesions of the amygdaloid complex. J. Neurosci. 21, 8238-8246.

McEwen, B.S., 2003. Mood disorders and allostatic load. Biol. Psychiatry 54, 200-207. McQuay, H.J., Tramèr, M., Nye, B.A., Carroll, D., Wiffenb, P.J., Moore, R.A., 1996. A systematic review of antidepressants in neuropathic pain. Pain 68, 217-227.

Meguro, R., Lu, J., Gavrilovici, C. Poulter M.O. 2004. Static, transient and permanent organization of GABA receptor expression in calbindin-positive interneurons in response to amygdala kindled seizures. J. Neurochem. 91, 144-154.

Merskey, H., 1965. Psychiatric patients with persistent pain. J. Psychosom. Res. 9, 299-309.
Merskey, H., Bogduk, N. (Eds.), 1994. Classification of Chronic Pain: Descriptions of 688 Chronic Pain Syndromes and Definitions of Pain Terms, 2nd ed. IASP Press, 689 Seattle.

Mesquita, A.R., Tavares, H.B., Silva, R., Sousa, N., 2006. Febrile convulsions in developing 691 rats induce a hyperanxious phenotype later in life. Epilepsy Behav. 9, 401-406. 692

Miller, M.W., Nowakowski, R.S., 1988. Use of bromodeoxyuridine-immunohistochemistry 693 to examine the proliferation, migration and time of origin of cells in the central 694 nervous system. Brain Res. 457, 44-52.

Mini, A., Rau, H., Montoya, P., Palomba, D., Birbaumer, N., 1995. Baroreceptor cortical 696 effects, emotions and pain. Int. J. Psychophysiol. 19, 67-77.

Mullen, R.J., Buck, C.R., Smith, A.M., 1992. NeuN, a neuronal specific nuclear protein in 698 vertebrates. Development 116, 201-211.

Neugebauer, V., Li, W., 1992. Processing of nociceptive mechanical and thermal 700 information in central amygdala neurons with knee-joint input. J. Neurophysiol. 87, 701 103-112.

Neugebauer, V., Li, W., Bird, G.C., Bhave, G., Gereau IV, R.W., 2003. Synaptic plasticity in 703 the amygdala in a model of arthritic pain: differential roles of metabotropic 704 glutamate receptors 1 and 5. J. Neurosci. 23, 52-63. 705

Neugebauer, V., Li, W., Bird, G.C., Han, J.S., 2004. The amygdala and persistent pain. 706 Neuroscientist 10, 221-234

Parent, J.M., Vexler, Z.S., Gong, C., Derugin, N., Ferriero, D.M., 2002. Rat forebrain 708 neurogenesis and striatal neuron replacement after focal stroke. Ann. Neurol. 52, 709 802-813.

Paxinos, G., Watson, C., 1998. The Rat Brain in Stereotaxic Coordinates, Fourth Ed. 711 Academic Press, New York. 712

Pertovaara, A., 2000. Plasticity in descending pain modulatory systems. Prog Brain Res. 713 129, 231-242.

Porsolt, R.D., Bertin, A., Jalfre, M., 1977. Behavioural despair in mice: a primary screening 715 test for antidepressants. Arch. Int. Pharmacodyn. Ther. 229, 327-336. 716

Porsolt, R.D., Anton, G., Blavet, N., Jalfre, M., 1978. Behavioural despair in rats: a new 717 model sensitive to antidepressant treatments. Eur. J. Pharmacol. 47, 379-391. 718

Porreca, F., Ossipov, M.H., Gebhart, G.F., 2002. Chronic pain and medullary descending 719 facilitation. Trends Neurosci. 25, 319-325

Rasmussen, P.V., Sindrup, S.H., Jensen, T.S., Bach, F.W., 2004. Symptoms and signs in 721 patients with suspected neuropathic pain. Pain 110, 461-469. 722

Reeves, S., Helman, L., Allison, A., Israel, M., 1989. Molecular cloning and primary 723 structure of human glial fibrillary acidic protein. Proc. Natl. Acad. Sci. 86, 5178-5182. 724

Rietze, R., Poulin, P., Weiss, S., 2000. Mitotically active cells that generate neurons and 725 astrocytes are present in multiple regions of the adult mouse hippocampus. 726 J. Comp. Neurol. 424, 397-408.

Ren, K., Dubner, R., 1996. Enhanced descending modulation of nociception in rats with 728 persistent hindpaw inflammation. J. Neurophysiol. 76, 3025-3037.

eynolds, B.A., Weiss, S., 1992. Generation of neurons and astrocytes from isolated cells 730 of the adult mammalian central nervous system. Science 255, 1707-1710.

Rhudy, J.L., Meagher, M.W., 2000. Fear and anxiety: divergent effects on human pain 732 thresholds. Pain $84,65-75$.

Schaible, H.G., Neugebauer, V., Cervero, F., Schmidt, R.F., 1991. Changes in tonic 734 descending inhibition of spinal neurons with articular input during the develop- 735 ment of acute arthritis in the cat. J. Neurophysiol. 66, 1021-1032.

Shim, B., Kim, D.W., Kim, B.H., Nam, T.S., Leem, J.W., Chung, J.M., 2005. Mechanical and 737 heat sensitization of cutaneous nociceptors in rats with experimental peripheral 738 neuropathy. Neuroscience 132, 193-201.

Sousa, N., Almeida, O.F.X., Wotjak, C.T., 2006. A hitchhiker's guide to behavioral analysis 740 in laboratory. Rodents Genes. Brain Behav. 5 (Suppl. 2), 5-24.

Steiner, B., Kronenberg, G., Jessberger, S., Brandt, M.D., Reuter, K., Kempermann, G., 742 2004. Differential regulation of gliogenesis in the context of adult hippocampal 743 neurogenesis in mice. Glia 46, 41-52.

Strakowski, S.M., DelBello, M.P., Sax, K.W., Zimmerman, M.E., Shear, P.K., Hawkins, J.M., 745 Larson, E.R., 1999. Brain magnetic resonance imaging of structural abnormalities in 746 bipolar disorder. Arch. Gen. Psychiatry 56, 254-260. 747

Takemura, N.U, 2005. Evidence for neurogenesis within the white matter beneath the 748 temporal neocortex of the adult rat brain. Neuroscience 134,121-132. $\quad 749$

Tal, M., Bennett, G.J., 1994. Extra-territorial pain in rats with a peripheral mononeuropathy: 750 mechano-hyperalgesia and mechano-allodynia in the territory of an uninjured nerve. 751 Pain 57, 375-382.

Taub, A., 1982. Opioid analgesic in the treatment of chronic intractable pain of non- 753 neoplastic origin. In: Kitahata, L.M., Colllins, J.G. (Eds.), Narcotic Analgesics in 754 Anesthesiology. Williams \& Wilkins, Baltimore/London, pp. 199-208.

Tebartz van Elst, L., Woermann, F., Lemieux, L., Trimble, M.R., 2000. Increased amygdala 756 volumes in female and depressed humans. A quantitative magnetic resonance 757 imaging study. Neurosci. Lett. 281, 103-106.

Tershner, S.A., Helmstetter, F.J., 2000. Antinociception produced by mu opioid receptor 759 activation in the amygdala is partly dependent on activation of mu opioid and 760 neurotensin receptors in the ventral periaqueductal gray. Brain Res. 865, 17-26. 761

Vanegas, H., Schaible, H.G., 2004. Descending control of persistent pain: inhibitory or 762 facilitatory? Brain Res. Rev. 46, 295-309.

Van Kampen, J.M., Hagg, T., Robertson, H.A., 2004. Induction of neurogenesis in the 764 adult rat subventricular zone and neostriatum following dopamine D receptor 765 stimulation. Eur. J. Neurosci. 19, 2377-2387.

Watkins, L.R., Maier, S.F., 2002. Beyond neurons: evidence that immune and glial cells 767 contribute to pathological pain states. Physiol. Rev. 82, 981-1011.

Weiss, S., Reynolds, B.A., Vescovi, A.L., Morshead, C., Craig, C.G., van der Kooy, D., 1996. 769 Is there a neuronal stem cell in the mammalian forebrain? Trends Neurosci. 19, 770 387-393.

Willoughby, S.G., Hailey, B.J., Mulkana, S., Rowe, J., 2002. The effect of laboratory- 772 induced depressed mood state on responses to pain. Behav. Med. 28, 23-31. 
Yoshimi, K., Ren, Y.R., Seki, T., Yamada, M., Ooizumi, H., Onodera, M., Saito, Y., Murayama, S., Okano, H., Mizuno, Y., Mochizuki, H., 2005. Possibility for neurogenesis in substantia nigra of Parkinsonian brain. Ann. Neurol. 58, 31-40.

Zelman, D.C., Howland, E.W., Nichols, S.N., Cleeland, C.S., 1991. The effects of induced mood on laboratory pain. Pain 46, 105-111.
Zimmermann, M., 1983. Ethical guidelines for investigations of experimental pain in 779 conscious animals. Pain 16, 109-110.

Zhao, M., Momma, S., Delfani, K., Carlen, M., Cassidy, R.M., Johansson, C.B., Brismar, H., 781 Shupliakov, O., Frisen, J., Janson, A.M., 2003. Evidence for neurogenesis in the adult 782 mammalian substantia nigra. Proc. Natl. Acad. Sci. U. S. A. 00, 7925-7930. 